\title{
Plants Utilise Ancient Conserved Peptide Upstream Open Reading Frames as Environmental Sensors
}

\author{
Barry Causier ${ }^{1}$, Tayah Hopes ${ }^{1}$, Mary McKay ${ }^{1}$, Zachary Paling ${ }^{1}$, and Brendan Davies ${ }^{1}$ \\ ${ }^{1}$ University of Leeds
}

October 31, 2021

\begin{abstract}
The regulation of protein synthesis plays an important role in growth and development in all organisms. Upstream open reading frames (uORFs) are commonly found in eukaryotic mRNA transcripts and typically attenuate the translation of associated downstream main ORFs (mORFs). Conserved peptide uORFs (CPuORFs) are a rare subset of uORFs, some of which have been shown to conditionally regulate translation by ribosome stalling. Here we identify three Arabidopsis CPuORFs of ancient origin that regulate translation of any downstream ORF, in response to agriculturally significant environmental signals: heat stress and water limitation. We provide evidence that different sequence classes of CPuORF stall ribosomes during different phases of translation and show that plant CPuORFs act as environmental sensors that can be utilised as inducible regulators of translation with broad application.
\end{abstract}

\section{Hosted file}

PCE CPuORF RESUB v1_26.10.21.doc available at https://authorea.com/users/411212/articles/ 543745-plants-utilise-ancient-conserved-peptide-upstream-open-reading-frames-asenvironmental-sensors 